\title{
Mediação de conflito: soluções propostas em atendimento a casos de violência contra a pessoa idosa
}

\author{
Conflict mediation: proposed solutions to deal with cases of violence against older people
}

Neuza Moreira de Matos' 1 (D)

Mariana Campos Braz² (D) Emanuelle de Oliveira Albernaz ${ }^{3}$ (D) Barbara Barbosa de Sousa ${ }^{4}$ ID Hudson Azevedo Pinheiro 5 ID Débora Thaís Timóteo Ferreirab ${ }^{\text {ID }}$

\section{Resumo}

Objetivo: Descrever os motivos da violência contra pessoas idosas e as soluções propostas na mediação de conflito em um ambulatório especializado em geriatria e gerontologia do Distrito Federal, Brasil, entre os anos de 2008 a 2018. Método: Estudo retrospectivo, documental, descritivo, com abordagem quantitativa, desenvolvido através da análise de informações obtidas em livros-ata da unidade, com registro de reuniões de mediação de conflito de casos de violência contra a pessoa idosa. A coleta abrangeu os motivos de violência contra a pessoa idosa e as soluções propostas na mediação de conflitos. Resultado: Foram analisados 111 casos. Os principais motivos da violência foram: sobrecarga do cuidador principal (77,4\%); filhos acharem que os pais idosos eram capazes de se cuidarem sozinhos (27\%); ressentimento dos filhos para com o idoso (24,3\%); e desconhecimento da doença do idoso $(14,4 \%)$. As principais soluções propostas foram: acompanhamento regular com médico (82,8\%), assistente social e/ou psicólogo (58,5\%); comprometimento de todos os filhos com a divisão dos cuidados e despesas dos pais idosos (52,2\%); introdução da pessoa idosa em atividades sociais da comunidade (27\%); e contratação de um cuidador formal (24,3\%). Conclusão: A sobrecarga do cuidador foi o principal motivo de conflito encontrado e as propostas apontadas se relacionavam a maior necessidade de cuidados em saúde com a pessoa idosa e seu cuidador, acerca disto, percebeu se a importância da disponibilidade de uma equipe multidisciplinar frente as

\section{Palavras-chave: Maus tratos ao idoso. Violência. Negociação. Cuidadores.}

1 Policlínica de Taguatinga, departamento de Geriatria e Gerontologia. Secretaria de Saúde (SES - DF). Brasília, DF, Brasil.

2 Hospital de Base, Instituto de Gestão Estratégica do Distrito Federal (IGESDF), departamento de Unidade de Internação. Brasília, DF, Brasil.

3 Serviço Social do Comércio (SESC), departamento de Educação em saúde. Brasília, DF, Brasil.

4 Universidade Católica de Brasília (UCB), departamento de Enfermagem. Brasília, DF, Brasil.

5 Policlínica de Taguatinga, departamento de Geriatria e Gerontologia. Secretaria de Saúde (SES - DF). Brasília, DF, Brasil.

6 Policlínica de Taguatinga, departamento de Geriatria e Gerontologia. Secretaria de Saúde (SES - DF). Brasília, DF, Brasil.

Os autores declaram não haver conflito na concepção deste trabalho.

Não houve financiamento para a execução deste trabalho. 
situações de violência. A mediação de conflito permitiu o estabelecimento de estratégias reais e direcionadas para alcance de resultados frente aos casos de violência.

\section{Abstract}

Objective: To describe the reasons for violence against older people and the solutions proposed for conflict mediation in an outpatient clinic specialized in geriatrics and gerontology in the Federal District, Brazil, between 2008 and 2018. Method: A retrospective, documentary, descriptive study with a quantitative approach developed with the analysis of information obtained in the unit's minutes books via the records of conflict mediation meetings in cases of violence against older people. The collection covered the reasons for violence against older people and the solutions proposed for conflict mediation. Result: We analyzed 111 cases. The main reasons for the violence were main caregiver burden (77.4\%), children thinking that their older parents were able to take care of themselves $(27 \%)$, resentment of children towards their older parents $(24.3 \%)$, and being unaware of the older person's disease (14.4\%). The main solutions proposed were regular follow-up with a doctor $(82.8 \%)$, social worker and/or psychologist $(58.5 \%)$, the commitment of all children in sharing care and expenses of their older parents $(52.2 \%)$, introducing the older person to social activities in the community $(27 \%)$, and hiring a formal caregiver (24.3\%). Conclusion: The caregiver burden was the main cause for conflict found, and the proposals identified were related to the greater need for health care for the older person and their caregiver. In this regard, the importance of a multidisciplinary team available in situations of violence was perceived. Conflict mediation made it possible to establish real and targeted strategies to achieve results in cases of violence.

\section{INTRODUÇão}

O envelhecimento da população traz consigo desafios para a saúde pública, na qual novas demandas e necessidades específicas desse grupo populacional estão a cada dia mais presentes nos serviços de saúde ${ }^{1}$. Envelhecer envolve perda gradativa da funcionalidade, podendo ocorrer declínio cognitivo, maior dependência global e, frequentemente, presença de doenças crônicas. Além de todas essas características comuns, esse grupo etário está vulnerável, ainda, ao fenômeno da violência ${ }^{2}$.

A violência contra a pessoa idosa é definida pela Organização Mundial de Saúde (OMS) 3 como qualquer ação, única ou repetida, ou ainda, a omissão de providência apropriada, ocorrida dentro de uma relação em que haja expectativa de confiança, que provoque danos e/ou aflição à vítima, pode ser classificada como: agressão física, psicológica, sexual, financeira, negligência e abandono ${ }^{4}$.
No Brasil, 28\% dos lares possuem pelo menos um idoso, e os principais agressores são os filhos e os cônjuges ${ }^{5,6}$. A estrutura da família contemporânea, na qual há a inserção da mulher no mercado de trabalho, menor número de filhos e maior número de divórcios contribuem para a marginalização do idoso e ocorrência de violência ${ }^{2,5}$, em contrapartida, a família representa a principal rede de apoio da pessoa idosa, sendo uma instituição concreta de referência, conforme a legislação brasileira? ${ }^{7}$

Face ao exposto, a complexidade do fenômeno da violência tem requisitado avanços frente modelos de assistência em saúde estereotipados, fragmentados e pouco eficazes. Verifica se a necessidade de abordagens em equipe, com disposição intersetorial e articulada, que permita a detecção e notificação da violência, além do devido acolhimento e atendimento interdisciplinar, com vistas a interromper ou minimizar situações conflituosas ${ }^{8,9}$.

A mediação de conflitos é uma técnica não jurídica, eficaz e ainda incipiente no Brasil. Realiza-se
Keywords: Elder Abuse. Violence. Negotiating. Caregivers. 
a partir de reuniões com participação de familiares e demais pessoas envolvidas no cuidado da pessoa idosa, podendo ser conduzidas por profissionais da saúde de diversas áreas, se houver capacitação para tal. A abordagem possui a finalidade de identificar os papéis de cada indivíduo na rede de apoio do idoso, traçar as dificuldades apontadas pelos participantes e articular compromissos de cuidado ${ }^{6,10}$.

O método de mediação baseia se na identificação das motivações que ocasionaram a violência para, a partir do diálogo e mútua cooperação entre as partes, traçar possíveis meios de resolutividade do conflito, a fim de não apenas extinguir situações violentas cometidas contra a pessoa idosa, mas prevenir novos agravos, minimizar os riscos e pactuar acordos de forma positiva $a^{6,710}$.

A análise dos dados obtidos por meio da mediação de conflitos oferece direcionamento para novos estudos e aplicabilidade na prática assistencial em saúde. Observa-se, ainda, que são incipientes as produções científicas acerca da mediação de conflitos e seus resultados. Desta forma, este estudo teve como objetivo descrever os motivos da violência contra a pessoa idosa, assim como as soluções propostas nas reuniões de mediação de conflitos entre os anos de 2008 a 2018, em um ambulatório especializado em geriatria e gerontologia do Distrito Federal (DF), Brasil.

\section{MÉTODO}

Estudo retrospectivo, documental-descritivo, com abordagem quantitativa, desenvolvido a partir da análise de informações colhidas de livros-ata, no qual estão contidos relatórios referentes a reuniões de mediação de conflitos de casos de violência contra pessoas idosas. Os atendimentos ocorreram entre os anos de 2008 e 2018 em um ambulatório especializado em saúde geriátrica e gerontológica no Distrito Federal (DF), Brasil. Esse ambulatório disponibiliza de uma equipe interdisciplinar especializada em atendimento à pessoa idosa, sendo o único de assistência ambulatorial integrada referenciado a esse contingente populacional na cidade.

Os casos captados e encaminhados na unidade se caracterizavam por situações específicas, como: idosos dependentes desacompanhados; idosos e cuidadores sem entendimento sobre o estado de saúde do idoso; idosos com declínio cognitivo importante em situação de negligência; idosos que relataram exploração financeira por parte dos familiares ou outros cuidadores; idosos em estado de abandono; idosos em suspeita de violência física e psicológica; dentre outros. Essas situações eram relatadas pelo próprio idoso, pelo familiar, por algum conhecido, pelo cuidador formal do idoso ou por qualquer pessoa da comunidade, inclusive profissional da saúde durante prestação de atendimento.

Qualquer integrante da equipe de saúde poderia encaminhar um relatório do caso ao serviço social, registrando a situação de violência ou suspeita de violência. As reuniões familiares ocorriam uma vez por semana, em data pré definida após acionamento, pelo assistente social, dos familiares e demais pessoas envolvidas no cuidado ao idoso para comparecer a unidade de saúde.

Os mediadores do conflito (assistente social, enfermeiro, psicólogo ou outro profissional da equipe) conduziam a reunião de mediação, quando presentes no mínimo dois profissionais de saúde. As atas eram lavradas em campo livre, geralmente, pelo assistente social, que por sua vez, também realizava a notificação da violência e seguimento dos casos e resultados alcançados.

Os critérios de inclusão utilizados foram: casos de idosos atendidos pela unidade especializada, possuir 60 anos ou mais e ter registro na ata de reuniões de mediação de conflitos.

As atas de reuniões registradas entre o ano de 2008 a 2018 foram analisadas de forma independente, por três pesquisadores, devidamente treinados, no período entre junho de 2018 a janeiro de 2019. Foram coletadas informações abertas quanto: motivos e propostas de soluções. As divergências ocorridas devido limitações encontradas nos registros eram discutidas em conjunto entre os seis pesquisadores. Os dados foram analisados com base na síntese de todas as reuniões realizadas em cada caso de violência, sendo acrescidos novas informações e resultados encontrados posteriormente quando ocorria uma segunda reunião. 
Nos livros-ata, constavam 143 casos, com uma ou mais reuniões familiares por caso, sendo excluídos as situações em que não houve violência e/ou atendimento de indivíduos com menos de 60 anos de idade, totalizando 111 casos na amostra pesquisada.

Para a análise dos dados, foi feita uma análise estatística descritiva, sendo contabilizados os dados relativos aos motivos da violência e as soluções propostas (pactuadas) para resolução dos casos de violência contra a pessoa idosa e construído dois quadros com a frequência absoluta e relativa das respostas.

Este estudo foi aprovado pelo Comitê de Ética da Fundação de Ensino e Pesquisa em Ciências da Saúde (FEPECS) mediante Parecer no 1.798.579, de
29 de outubro de 2016, com realização de adendo para dar-se continuidade do estudo nos anos de 2017 e 2018. Foi desenvolvido ainda, conforme as Resoluções no 466/2012 e no 510/2016 do Conselho Nacional de Saúde.

\section{RESULTADOS}

Foram analisados 111 casos registrados no livro ata. Dentre os 10 anos de registro, observou-se um decréscimo no número de ocorrências de violência identificados na unidade conforme apresenta a figura 1. Ressalta-se que entre os anos de 2015 a 2018 houve períodos de indisponibilidade de profissional habilitado (assistente social), reduzindo consequentemente o número de atendimentos de mediação de conflitos neste período.

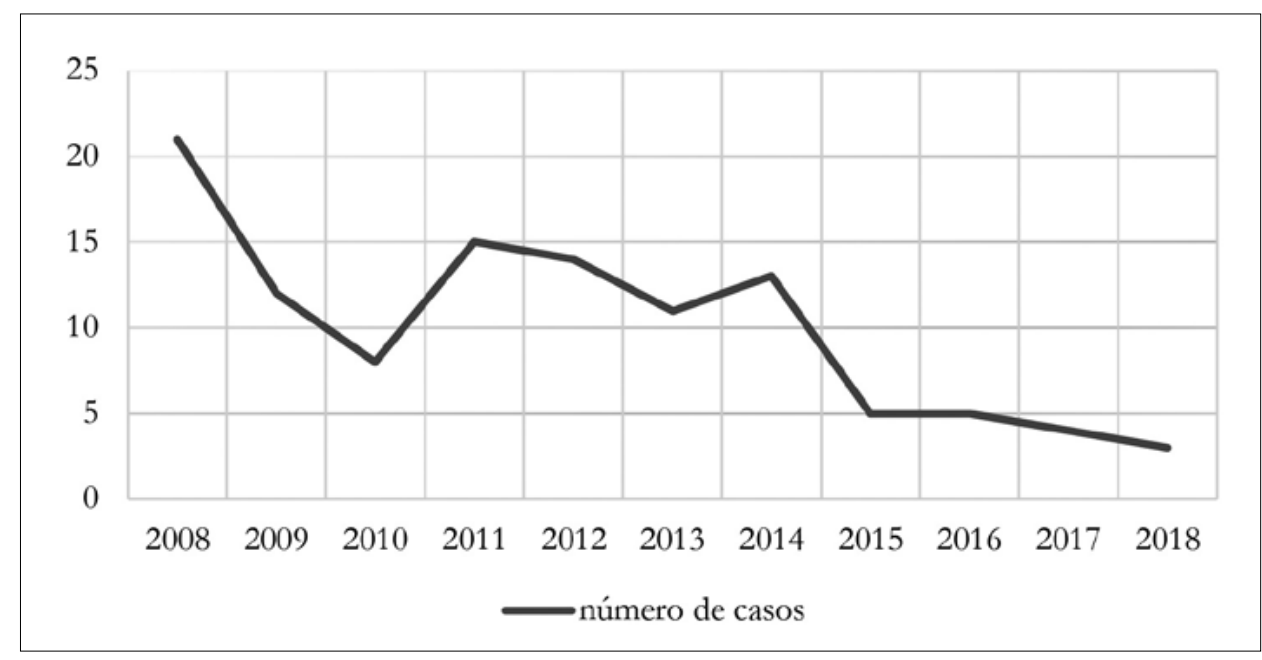

Figura 1. Número de casos de violência registrados entre 2008 e 2018 em um ambulatório especializado de geriatria e gerontologia (N=111). Distrito Federal, DF, Brasil (2008-2018).

Acerca dos idosos vítimas de violência, $72 \%$ eram mulheres, a faixa etária predominante foi de 81 a 90 anos $(45 \%)$ e 71 a 80 anos (39\%), $5 \%$ eram nonagenários. A maioria dos idosos residiam em moradia própria, com renda de um salário mínimo $(46 \%)$. No que concerne as comorbidades identificadas, $54 \%$ dos idosos tinham quadro demencial, $31 \%$ hipertensão arterial sistêmica e 13\% diabetes mellitus. Dos idosos com demência, 32\% dos indivíduos envolvidos no cuidado, desconheciam sintomas da doença.

Os filhos foram os principais agressores identificados (72\%), sendo $62 \%$ dos agressores do sexo masculino e $38 \%$ do sexo feminino. Acerca da faixa etária do agressor, houve predominância entre 51 a 60 anos (37\%) e idade entre 41 a 50 anos (30\%). 
Em relação ao tipo de violência sofrida, observou-se maior prevalência da negligência (56\%), seguida da violência psicológica (29\%), violência física $(8 \%)$ e abuso financeiro $(6 \%)$. A ocorrência de negligência associada ao abandono representou $21 \%$ dos casos.

Conforme apresentando na tabela 1 , identificouse a sobrecarga do cuidador principal como o motivo mais comum $(77,4 \%)$. Outro achado frequente foi a falta de conhecimento acerca da capacidade funcional do idoso, levando à negligência por acreditar que ele realizaria suas atividades sem supervisão ou intervenção, representando $27 \%$ da amostra.

Os dados alcançados nos casos de violência praticados por filhos demonstraram que 24\% possuíam ressentimentos para com a pessoa idosa ou fragilidade do laço afetivo construído ao longo da história de ambos, resultando, assim, em frequentes situações de conflitos e atos de violência. Outros $27 \%$ acreditavam que os pais idosos eram capazes de realizar suas atividades sozinhos.

Paralelamente a esse dado anterior, 6,3\% dos cuidadores não prestavam cuidados contínuos a pessoa idosa quando este, pela sua condição de saúde, necessitava de auxílio e cuidados 24h/dia.
Acerca dos idosos com demência, observou-se que, em 14,4\% dos casos, os cuidadores desconheciam o diagnóstico do idoso ou não possuíam nenhum entendimento sobre a doença, comportamentos comuns e como se portar frente às situações consequentes desse quadro.

Entre as motivações relacionadas ao cuidador principal, 6,3\% possuíam quadro de depressão e 4,5\% apresentavam dependência ao álcool.

Quanto às soluções propostas durante a reunião de mediação de conflito, pode se observar na Tabela 2 que, para cada caso analisado, houve mais de uma solução acordada na mediação.

Em grande parcela (92 casos), recomendou-se o acompanhamento regular com a equipe médica disponível na unidade de atendimento. Mais da metade dos casos analisados necessitaram ainda de encaminhamento e acompanhamento pelo serviço de assistência social e psicologia (58,6\%).

Dos 111 casos, em 38 houve acordo de cooperação entre os filhos durante reunião com a equipe e familiares. Em 27 casos, decidiu-se optar pela contratação de um cuidador formal. A inserção do idoso em atividades na comunidade representou $27 \%$ das soluções propostas aos casos.

Tabela 1. Motivos da violência contra a pessoa idosa no ambulatório especializado de geriatria e gerontologia do Distrito Federal, DF, Brasil (2008-2018).

\begin{tabular}{ll}
\hline Motivos da violência contra a pessoa idosa & Número de Casos (\%) \\
\hline Sobrecarga do cuidador principal. & $86(77,4 \%)$ \\
\hline Filhos acreditavam que os pais idosos eram capazes de se cuidarem sozinhos. & $30(27 \%)$ \\
\hline Ressentimento dos filhos para com o idoso ou fragilidades dos laços afetivos. & $27(24,3 \%)$ \\
\hline Desconhecimento acerca da doença do idoso (casos de demência). & $16(14,4 \%)$ \\
\hline $\begin{array}{l}\text { Desinteresse do cuidador na supervisão da medicação do idoso e/ou descaso } \\
\text { com doenças crônicas. }\end{array}$ & $15(13,5 \%)$ \\
\hline Alterações comportamentais do cuidador. & $8(7,2 \%)$ \\
\hline Cuidador principal com quadro de depressão. & $7(6,3 \%)$ \\
\hline Desconhecimento acerca da necessidade de cuidados contínuos $(24 \mathrm{~h} /$ dia) & $7(6,3 \%)$ \\
\hline Cuidador principal com dependência alcoólica. & $5(4,5 \%)$ \\
\hline
\end{tabular}

Fonte: Elaborada pelos autores. 
Tabela 2. Soluções propostas pela mediação de conflito no ambulatório especializado de geriatria e gerontologia do Distrito Federal, DF, Brasil (2008-2018).

\begin{tabular}{lc}
\hline Soluções propostas pela mediação de conflito & Número de casos (\%) \\
\hline $\begin{array}{l}\text { Acompanhamento regular com médico (médico da família ou geriatra) quanto à } \\
\text { evolução das doenças, especialmente da demência, e ao controle de doenças crônicas. }\end{array}$ & $92(82,8 \%)$ \\
\hline $\begin{array}{l}\text { Acompanhamento regular do idoso com assistente social e/ou psicólogo. } \\
\text { Comprometimento de todos os filhos com a divisão dos cuidados e/ou das } \\
\text { despesas dos pais idosos. }\end{array}$ & $55(58,5 \%)$ \\
\hline $\begin{array}{l}\text { Introdução do idoso em atividades sociais da comunidade. } \\
\text { Contratação de cuidador formal. }\end{array}$ & $30(27 \%)$ \\
\hline
\end{tabular}

Fonte: Elaborada pelos autores.

Houve o encaminhamento do cuidador para acompanhamento de serviço de saúde que atendesse a sua demanda (psicólogo, psiquiatra e médico), em 12 casos, nos quais se detectou depressão grave e alcoolismo.

Houve ainda, em 18 casos, a inserção dos idosos com demência em grupos de terapia cognitiva, dirigidos por terapeuta ocupacional e enfermeiro, além do desenvolvimento de atividades manuais (artesanato) disponíveis no ambulatório especializado.

\section{DISCUSSÃO}

As motivações relacionadas ao processo de violência contra a pessoa idosa variam, sendo possível observar a presença de mais de um motivo apontado em diversos casos do presente estudo. Dentre eles, ressalta-se a sobrecarga que o processo de cuidar impõe aos cuidadores, relatada em $77,4 \%$ dos casos. O estresse emocional prolongado está atrelado ao exercício de cuidar atribuído a apenas um indivíduo que, geralmente, não optou por desempenhar esse papel ou não possui treinamento adequado para exercê-lo ${ }^{11}$.

O alto grau de dependência do idoso para as atividades da vida diária (AVD) e atividades instrumentais da vida diária (AIVD) caracteriza maior risco para violência ${ }^{12}$ e maior índice de sobrecarga dos cuidadores, que, por sua vez, é fator desencadeante para a negligência assistencial da pessoa cuidada, com omissão de necessidades básicas a pessoa idosa ${ }^{11}$.
O impacto que esse cuidado contínuo gera pode ser atenuado promovendo a partilha com as demais redes de apoio desse idoso, seja nos arranjos familiares ou em grupos de suporte psicoeducativos. Sabe-se que o equilíbrio adequado entre a demanda de cuidados e o tempo ofertado de assistência pode produzir melhores resultados quando há preparo e devido treinamento desse cuidador ${ }^{11}$.

A figura do cuidador é representada em estudos como um familiar, do sexo feminino, com idade média de 53,9 anos, que reside no mesmo domicílio da pessoa idosa ${ }^{11,13}$.O residir no mesmo lar favorece a ocorrência de violência ${ }^{11}$, a qual, por sua vez, é camuflada na tentativa de preservar os laços ou justificada frente a sentimento de culpa por parte do idoso. Por vezes, a violência passa despercebida, vista como um padrão de comportamento ou estresse interpessoal do cuidador ${ }^{13,14}$.

Os ressentimentos dos filhos para com a pessoa idosa ou fragilidades dos laços afetivos foram motivações também encontradas nesta pesquisa (27\%). Outras razões apresentadas em diferentes estudos envolvem questões financeiras, conflito de interesses entre as gerações e interdependência entre os envolvidos ${ }^{12,15}$.

Nessas problemáticas, a mediação de conflito constitui um recurso essencial para restauração de relações e reflexão da realidade vivida. Estudo acerca da mediação familiar de Martins ${ }^{7}$ aborda a família atual como uma instituição social que não dispõe de informação e apoio para desempenho da tarefa de cuidar. Acerca disto, para uma abordagem 
resolutiva, faz se necessário um olhar voltado para o contexto social e todo processo em que os sujeitos estão inseridos ${ }^{7}$.

Dentre outras razões para violência detectadas neste estudo, em 14,4\% dos casos, havia desconhecimento acerca da demência e suas apresentações. Ao analisar fatores de risco para a violência, estudos relacionam a demência com o aumento em até quatro vezes de ocorrência de atos violentos contra a pessoa idosa ${ }^{12,16}$.

Idosos acometidos pela demência podem agir de forma agressiva e gerar no cuidador um ato violento recíproco ${ }^{12}$. O esclarecimento acerca das possíveis alterações comportamentais favorece um entendimento do cuidador e protege o idoso vulnerabilizado de danos e sofrimento. O profissional de saúde capacitado para o devido manejo e gerenciamento da doença torna-se imprescindível ${ }^{15}$.

Acerca dos cuidadores de idosos com demência, estudo recente detectou prevalência de depressão e síndrome do pânico em $8 \%{ }^{16}$. O adoecimento do cuidador também foi relatado nesta pesquisa, no qual houve registro de depressão em 6,3\% dos casos.

A oferta de cuidado a um indivíduo exige empenho biopsicossocial e estudos demonstram que a qualidade de vida do cuidador interfere diretamente na qualidade do cuidado desempenhado por ele. A figura do cuidador frequentemente passa sob um olhar desatento por parte dos profissionais de saúde e carece de apoio social. As relações interpessoais positivas e manifestações de afeição e amor são exemplos de recursos oferecidos por outros ao cuidador que contribuem para melhoria de sua qualidade de vida ${ }^{13,17}$.

Ao analisar outros fatores relacionados ao cuidador que contribuem para maior incidência de violência, Lino et al. ${ }^{15}$ identificaram o alcoolismo como variável importante, provocando o aumento de 3,8 vezes o risco de atos violentos. O uso abusivo do álcool pode ser identificado quando gera consequências sociais advindas do seu consumo.

A oferta de acompanhamento e tratamento desse cuidador se torna fundamental na interrupção de um hábito que gera conflitos, desgastes nas relações e sofrimento humano ${ }^{18}$. Neste estudo, o acompanhamento médico e psicológico do cuidador foi estabelecido como solução proposta, visto que, em cinco casos, houve relato de dependência alcoólica.

O desafio para identificação da violência perpetrada no ambiente familiar é, por vezes, mascarado como padrão de relacionamento comum. A despeito disto, percebeu-se a importância da capacitação contínua dos profissionais de saúde para escuta sensível e atenta dos idosos e cuidadores em cada atendimento.

Acerca da mediação de conflitos, pode-se detectar, através dela, importantes fragilidades sociais, financeiras e emocionais nos cuidadores, os quais carecem de suporte profissional e social, com escasso ou mesmo nenhum amparo e orientação para exercício do cuidar.

Uma possível limitação no estudo ocorreu quanto à baixa sistematização das perguntas para registro, pois a construção da ata não objetivava ser instrumento de pesquisa; desta forma, alguns dados da prática de mediação podem não ter sido relatados ou relatados com imprecisão. Sugere-se a construção de atas semi estruturadas a fim de não se perder informações relevantes.

\section{CONCLUSÃO}

A análise dos resultados obtidos pela mediação de conflitos mostra a necessidade de intervenções direcionadas ao cuidador principal, como ações de promoção de saúde física e mental, visto que a sobrecarga foi um achado frequente, atrelado ainda aos casos de depressão e dependência alcoólica encontrados. Acerca da falta de conhecimento sobre a doença do idoso, vale destacar a importância de treinamentos e capacitações das pessoas envolvidas no cuidado, afim de prevenir as formas de violência decorrentes de negligência. A disponibilidade de uma equipe multidisciplinar adquire caráter essencial no que concerne a atenção a pessoa idosa vítima de violência, visto que, entre as principais soluções propostas, houve grande demanda para atendimento do psicólogo, assistente social e médico geriatra disponíveis na unidade. 
A violência direcionada à pessoa idosa corresponde a um fenômeno multifatorial e a análise das motivações permite às entidades e profissionais de saúde traçarem planos de cuidados a fim de prevenir situações que gerem violência, viabilizando possibilidades reais e plausíveis de resolução conforme a situação apresentada.
Observa-se a grande necessidade de construção de novas iniciativas e metodologias de saúde voltados para o público idoso, visto que a assistência integral focalizada nas necessidades de cada indivíduo permite melhores resultados.

Editado por: Tamires Carneiro de Oliveira Mendes

\section{REFERÊNCIAS}

1. Veras RP, Oliveira M. Envelhecer no Brasil: a construção de um modelo de cuidado. Ciênc Saúde Colet. 2018;23(6):1929-36. Disponível em: https://doi. org/10.1590/1413-81232018236.04722018

2. Barros RLM, Leal MCC, Marques APO, Lins MEM. Violência doméstica contra idosos assistidos na atenção básica. Saúde Debate. 2019;43(122):793-804. Disponível em: https://doi.org/10.1590/0103-1104201912211

3. World Health Organization. Abuse of older people on the rise - 1 in 6 affected [Internet]. Geneva: WHO; 2017. Disponível em: https://www.who.int/en/newsroom/detail/14-06-2017-abuse-of-older-people-onthe-rise-1-in-6-affected.

4. Minayo MCS. Múltiplas faces da violência contra a pessoa idosa. Mais 60: Estud Envelhec. 2014;25(60):10-27. Disponível em: https://www. sescsp.org.br/files/edicao_revista/c31b6bcb-842a4b02-8a3c-cf781ab0d450.pdf.

5. Silva CFS, Dias CMSB. Violência contra idosos na família: motivações, sentimentos e necessidades do agressor. Psicol Ciênc Prof. 2016;36(3):637-52. Disponível em: https://doi.org/10.1590/19823703001462014.

6. Matos NM, Albernaz EO, Sousa BB, Braz MC, Vale MS, Pinheiro HA. Perfil do agressor de pessoas idosas atendidas em um centro de referência em geriatria e gerontologia do Distrito Federal, Brasil. Rev Bras Geriatr Gerontol. 2019;22(5):e190095. Disponível em: https://doi.org/10.1590/1981-22562019022.190095.

7. Martins ANE. Mediação familiar para idosos em situação de risco [Internet]. São Paulo: Blucher; 2017 [acesso em 06 set. 2018]. Disponível em: https://www. blucher.com.br/livro/detalhes/mediacao-familiarpara-idosos-em-situacao-de-risco-1294

8. Belasco AGS, Okuno MFP. Realidade e desafios para o envelhecimento. Rev Bras Enferm. 2019;72(Suppl2):1-10. Disponível em: https://doi. org/10.1590/0034-7167.2019-72suppl201.
9. Minayo MCS, Souza ER, Silva MMA, Assis SG. Institutionalizing the theme of violence within Brazil's national health system: progress and challenges. Ciênc Saúde Colet. 2018;23(6); $2007-$ 16. Disponível em: https://www.scielo.br/j/csc/a/ Q3kCPCWfBzqh8mzBnMhxmYj/?lang=pt.

10. Vale MS, Faleiros VP, Santos IB, Matos NM. Mediação de conflitos de violência intrafamiliar contra pessoas idosas: uma proposta não jurídica. Textos Contextos. 2015;14(1):104-14. Disponível em: https://doi.org/10.15448/1677-9509.2015.1.18168.

11. Nunes DP, Brito TRP, Duarte YAO, Lebrão ML. Cuidadores de idosos e tensão excessiva associada ao cuidado: evidências do Estudo SABE. Rev Bras Epidemiol. 2018;21(Suppl 2):e180020. Disponível em: https://doi.org/10.1590/1980-549720180020.supl.2.

12. Santos MAB, Moreira RS, Faccio PF, Gomes GC, Silva VL. Fatores associados à violência contra o idoso: uma revisão sistemática da literatura. Ciênc Saúde Colet. 2020;25(6):2153-75. Disponível em: https://doi.org/10.1590/1413-81232020256.25112018.

13. Jesus ITM, Orlandi AAS, Zazzetta MS. Sobrecarga, perfil e cuidado: cuidadores de idosos em vulnerabilidade social. Rev Bras Geriatr Gerontol. 2018;21(2):199-209. Disponível em: https://doi. org/10.1590/1981-22562018021.170155.

14. Silva AR, Sampaio LS, Reis LA, Sampaio TSO. Violência contra idosos: associação entre o gênero dos agressores e o tipo de violência. Id On Line Rev Multidiscipl Psic. 2017;11(38):1-9. Disponível em: https://doi.org/10.14295/idonline.v11i38.967.

15. Lino VTS, Rodrigues NCP, Lima IS, Athie S, Souza ER. Prevalência e fatores associados ao abuso de cuidadores contra idosos dependentes: a face oculta da violência familiar. Ciênc Saúde Colet. 2019;24(1):87-96. Disponível em: https://doi. org/10.1590/1413-81232018241.34872016. 
16. Silva ILC, Lima GS, Storti LB, Aniceto P, Formighieri PF, Marques S. Sintomas neuropsiquiátricos de idosos com demência: repercussões para o cuidador familiar. Texto \& Contexto Enferm. 2018;27(3):e3530017.

Disponível em: https://doi.org/10.1590/010407072018003530017.
17. Anjos KF, Boery RNSO, Pereira R, Pedreira LC, Vilela ABA, Santos VC, et al. Associação entre apoio social e qualidade de vida de cuidadores familiares de idosos dependentes. Ciênc Saúde Colet. 2015;20(5):1321-30. Disponível em: https://doi. org/10.1590/1413-81232015205.14192014.

18. Lopes APAT, Ganassin GS, Marcon SS, Decesaro MN. Abuso de bebida alcoólica e sua relação no contexto familiar. Estud Psicol. 2015;20(1):22-30. 4669.20150004. 